\title{
Evaluation of Feature Selection and Model Training Strategies for Object Category Recognition
}

\author{
Haider Ali* and Zoltan-Csaba Márton*
}

\begin{abstract}
Several methods for object category recognition in RGB-D images have been reported in literature. These methods are typically tested under the same conditions (which we can consider a "domain" in a restricted sense) such as viewing angles, distances to the object as well as lightening conditions on which they are trained. However, in practical applications one often has to deal with previously unseen domains.

In this paper, we investigate the effect of domain change on the performance of object category recognition methods. We use the public RGB-D Object Dataset from Lai et al. [1] for training, and for testing we introduce the DLR-RGB-D dataset, representing a similar, but different domain. The data present in both datasets holds various object instances grouped into general object categories. Object category detectors are trained using the objects of one domain and tested on the objects of the other domain. We then explored how do different 3D features perform when the model trained on the source domain is applied on the target domain, and evaluated two feature selection strategies.

In our experiments we show that a domain change can have significant impact on the model's accuracy, and present results for improving the results by increasing the variability of the objects in the training domain. Finally, we discuss the relevance of the descriptors and the properties they capture.

Index Terms - object categorization, cross-domain learning, feature selection, domain adaptation, RGBD object databases
\end{abstract}

\section{INTRODUCTION}

We consider the problem of domain change, as one of the major obstacles faced in practical adaptation of object categorization algorithms is that object instances used for testing are different than the training ones, and they might be captured under slightly different conditions than the ones used for building a training database. Domain in this context refers to properties of a dataset that are dependent on the data capturing process and conditions.

For RGB data, it has been shown that domain change severely affects recognizer performance, in spite of the fact that feature detectors and descriptors aim to be as invariant as possible. Therefore, we are continuing our previous work on categorizing high-variance RGB-D data [2], focusing here on increasing the robustness of the used classifiers by selecting the right features and augmenting the training dataset.

In order to evaluate the effect of relying on an online RGB$\mathrm{D}$ object database during category recognition performed in a different environment, we have used the largest available benchmark RGB-D dataset for training, and a new dataset (DLR-RGB-D) for testing. Our database contains 21 object

\footnotetext{
* Author names in alphabetical order (equal contribution).

The authors are with the Robotics and Mechatronics Center (RMC), German Aerospace Center (DLR), Oberpfaffenhofen, Germany \{Haider.Ali, Zoltan.Marton\}edlr.de
}

categories recorded on a table with Microsoft Kinect [3], and it is publicly available for the community, in order to support other benchmarking experiments. Using the 21 object categories that are common between the two sources, we train our classifier using the categories from one domain and see how that classifier performs on samples from the other domain. The main objective is to see how much robustness is provided by depth information against domain change.

We have used combinations of well known 3D features (VFH, ESF, PFH as summarized in [4]), and trained SVM classifiers. For feature selection we have used the minimum Redundancy Maximum Relevance (mRMR) and Maximal Relevance (MaxRel) feature selection methods [5]. The mRMR feature selection algorithm selects features with minimizing redundancy and takes into account their highest relevance to the target class. The MaxRel feature selection algorithm selects features with the consideration of highest relevance of features to the target class. We have tested our classifier on the RGB-D Dataset using 5-fold cross validation as well as on the DLR dataset, without adaptation, to highlight the difficulties encountered during cross-domain categorization. During our evaluations we investigated various combination of features as well as the amount of data used to train the classifier for optimizing performance as reported in the experimental section of the paper.

We report on the following contributions in this work:

- quantifying the generalizing power of object category recognition between datasets;

- evaluating the VFH, ESF and PFH features and their combinations (full and partial concatenation);

- analyzing the results given by features selection methods (mRMR and MaxRel);

- quantifying the effect of domain change from the RGBD (original) to the DLR (target) domain;

- improving the categorization accuracy obtained through adapting the training set.

In the following we will give an overview of the related approaches, then describe our work in sections III-V, present the experimental results in Section VI and discuss our findings in Section VII before finally concluding and discussing further research directions in VIII.

\section{RELATED WORK}

Object recognition has been an active area of research in computer vision. Object category recognition extends this concept to recognize classes of objects (chair, car, ...) instead of individual object instance detection. In a typical scenario for object recognition, training is performed on a 
subset of a Dataset and tested on the remaining subset. Recent challenges are to improve the robustness of the object recognition systems as well as their detection accuracy.

Several methods proposed in the literature for object recognition are based on the available textural information in RGB images data. The focus have been to propose different features based on the available textural information. For instance, [6] has proposed a real time multi-resolution object detection framework using Histograms of Oriented Gradient (HOG) features. A hierarchical region based object detection framework based on coherent probabilistic model has been proposed in [7]. Similarly, A region based object segmentation method using salient information (holistic properties of object shapes, geometric relationship of object boundaries) has been introduced by [8]. Another work [9] has proposed a learning framework of object detection and classification by concatenation of feature and context information using Support Vector Machine (Context-SVM).

In robotics and vision community, a major challenge is the real time object recognition and pose estimation for manipulation tasks on RGB-D (color+depth) data using sensors like Microsoft Kinect [3]. Lai et. al [10] has presented an object recognition framework for based on the image-level depth features using hierarchical kernel descriptors. Burrus et. al [11] have recently proposed a model based 3D shape reconstruction approach using classical histogram comparison for the task of pose estimation. Therefore, different methods have been proposed to adapt classifiers trained on one domain to the other domain.

A visual object recognition framework with domain adaption using web data has been introduced by Lai et. al [12]. They introduce a probabilistic exemplar-based method using SVM on Google 3D Warehouse and local Datasets. Bo et. al [13] have presented an unsupervised object recognition framework using hierarchical feature representations from RGB-D data. They have introduced dictionary learning mechanism to generate RGB-D depth and color image features. They have reported accuracy for category and instance recognition in comparison to their existing work and previously available approach (Convolutional K-Means descriptor [14]).

\section{RGB-D DLR OBJECT DATASET}

The RGB-D DLR Dataset is collected using by Microsoft Kinect [3]. The acquirement of each frame is performed by the Point Cloud Library (PCL) [15], using the OpenNI Grabber Framework. The Kinect is placed about one meter from the table where the objects to be sampled are placed. The data was taken with the Kinect mounted at $45^{\circ}$ above the horizontal. An example frame is shown in Figure 1, on which standard 3D object segmentation methods from PCL are used to extract the objects.

One revolution of each object was recorded, the rotation of the object was done manually rotating it around $5^{\circ}$ in each frame, and in some cases, depending on the shape of the object, the object was rotated in more than one axis. This procedure gives a total of 7893 RGB-Depth frames in

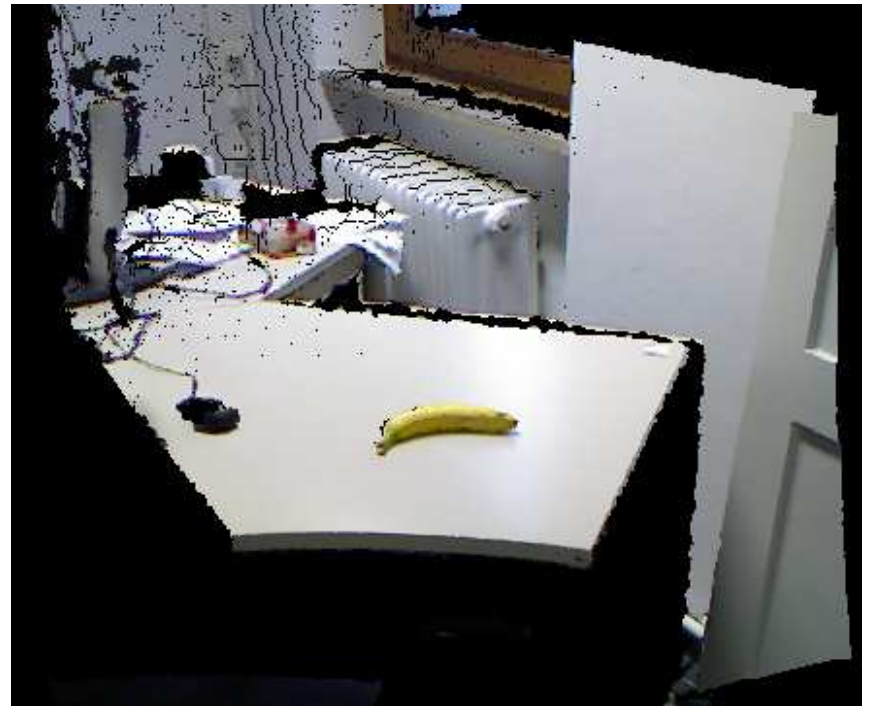

Fig. 1. An example of RGB-D DLR Dataset scene

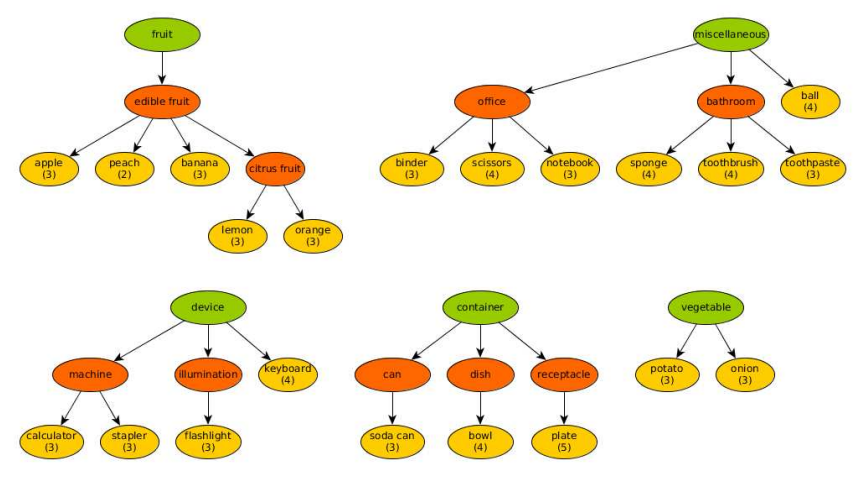

Fig. 2. RGB-D Object Dataset objects hierarchy. The number of instances in each leaf category is given in parentheses.

the DLR RGB-D Object Dataset. The DLR RGB-D Object Dataset contains 70 different objects in 21 categories, these categories are also included in RGB-D Dataset from Lai [1]. Figure 2 shows the categories and objects collected.

Once we have the cropped point cloud, To find the table and object, we assume that all the sampled objects are lying on the planar surface, then we perform RANSAC plane fitting to find the table plane and take points that lie above it to be the object. To remove the outlier in segmented objects, we have applied Radius Outlier Removal filter PCL [15]. The segmentation results are shown in Figure 3.

The resulting DLR dataset can be found at: http:// dlr.de/rmc/rm/de/staff/haider.ali/

\section{FEATURE EXTRACTION}

As we found in our previous work [16] that geometric features perform better for categorization of previously unknown object instances, we focused here on three promising global 3D object descriptors that were evaluated and described in [4]: the Point Feature Histograms (PFH), the Viewpoint Feature Histogram (VFH), and the Ensemble of Shape Functions (ESF). 


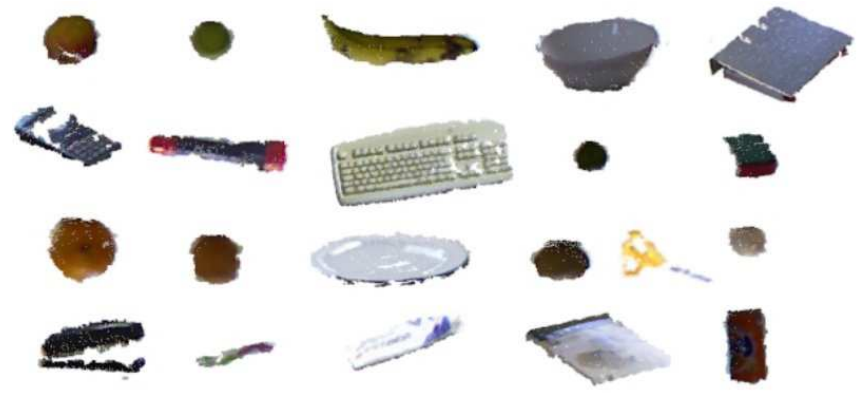

Fig. 3. Examples of 21 object categories from the RGB-D DLR Dataset.

All three capture various 3D surface properties, and have a high number of dimensions: 125 for $\mathrm{PFH}, 308$ for $\mathrm{VFH}$ and 640 for ESF. We used the tools available in PCL to process the input point clouds, namely to estimate surface normals, subsample the points s.t. the resolution is around $1 \mathrm{~cm}$, and to compute the feature descriptors for each input file. The feature matrices were concatenated, thus creating a descriptor of length 1073, as such concatenations provide a good way to fuse the information from different sources [17], [18]. However, some tests were performed using the individual features separately as well.

\section{Multi-Class Object Classification}

We used multi-class Support Vector Machine (SVM) classifiers with a linear kernel [19]. A linear kernel was chosen because it is faster to train and it gives comparable results to RBF Kernel for such large number of features/samples.

In order to investigate which positions in the high dimensional feature descriptors hold the most discriminative power, we used the mRMR and MaxRel algorithms [5] for the identification of best 500 features. We have then selected the best $50,100,150, \ldots$, and 500 as well as all the features for training different models.

For different combination of features descriptors (VFH, ESF and PFH) using mRMR and MaxRel, we have repeated the given sequence of feature selection separately. In Addition to that we also combine the top scoring of each of the feature types (VFH, ESF, PFH) Top 50, Top 100 and compare with the globally best 150 and 300 selected by mRMR and MaxRel.

\section{EXPERIMENTS AND RESULTS}

In this section we will present the results of our evaluations. All experiments, where only a subset of the feature descriptor vectors' dimensions were used, were performed twice, once using the feature ranking provided by mRMR and then using the ones by MaxRel. The two methods select the same top 500 features, but order them differently, as shown in Figure VII. The top 118 dimensions according to MaxRel are scored in the same order by mRMR, but the remaining ones are mingled.

First, we checked the 5-fold cross-validation results we obtained on the RGB-D dataset separately. The SVM parameters were selected such that they maximize this cross-

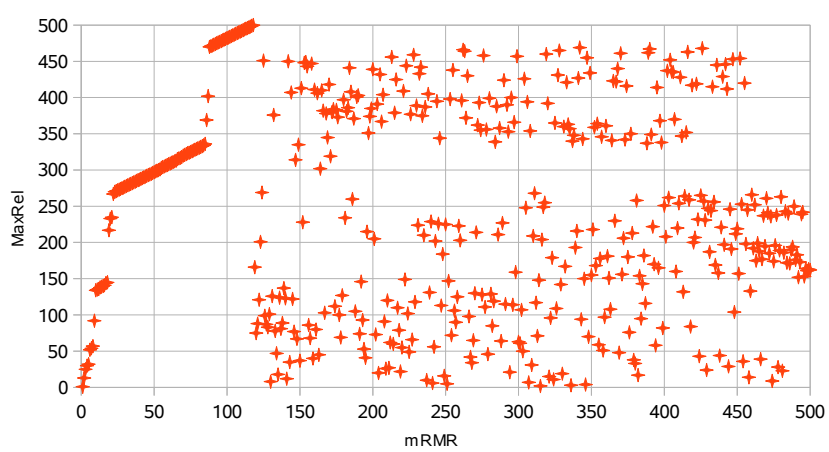

Fig. 4. Comparing the ordering of the best 500 feature dimensions by mRMR and MaxRel. The first 118 dimensions according to MaxRel are sorted in the same (relative) order by mRMR, but the remaining ones do not show any correlation between the two methods.

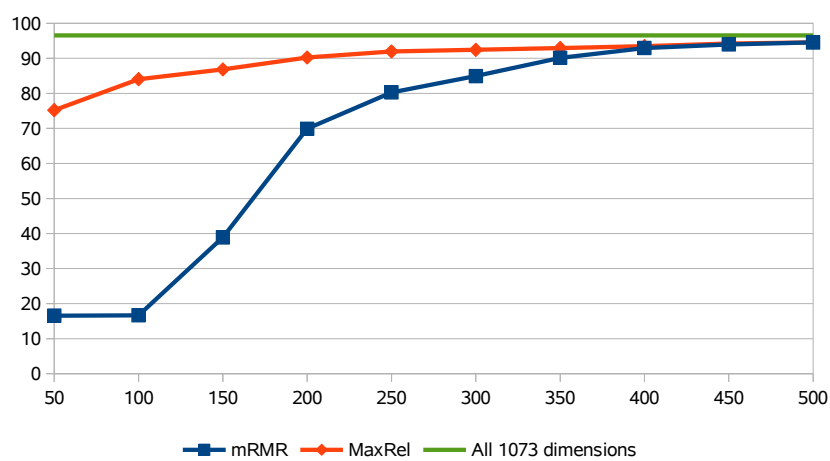

Fig. 5. Linear SVM 5-fold cross-validation accuracies on the RGB-D Object Dataset using the concatenation of all three descriptors. The top scoring feature dimensions according to mRMR and MaxRel were tested in steps of 50 up to 500, and compared to the case when every feature dimension is kept.

validation accuracy, and very good results were obtained, as shown in Figure 5.

Interestingly, contrary to our expectations based on [5], the feature vector dimensions scored higher by MaxRel performed in general much better than the ones scored highly by mRMR. This finding was confirmed in successive experiments, as we will show, suggesting that for the task of identifying the most important feature dimensions (and indirectly the object properties) the MaxRel method is more useful.

Looking at the features individually (Figure 6), we can see that a small subset of the ESF and PFH features already captures most of the variance between the object categories, while in the case of VFH the accuracy increases steadily as more and more dimensions are added. This means that a large portion of the information that PFH and ESF capture is redundant, or not too relevant for this categorization task. On the other hand, a small portion of them (between 100 and 150 dimensions) already has the same discriminative power as the full 308 dimensions of VFH.

We also evaluated the effect of selecting the top scoring 50 or 100 features from each descriptor and concatenating 


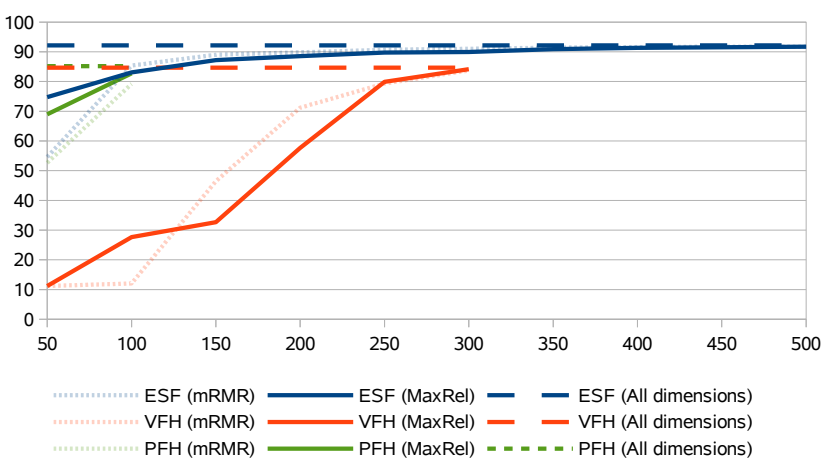

Fig. 6. Linear SVM 5-fold cross-validation accuracies on the RGB-D Object Dataset using the three descriptors separately. The top scoring feature dimensions according to mRMR and MaxRel were tested in steps of 50, and compared to the case when every feature dimension is kept.
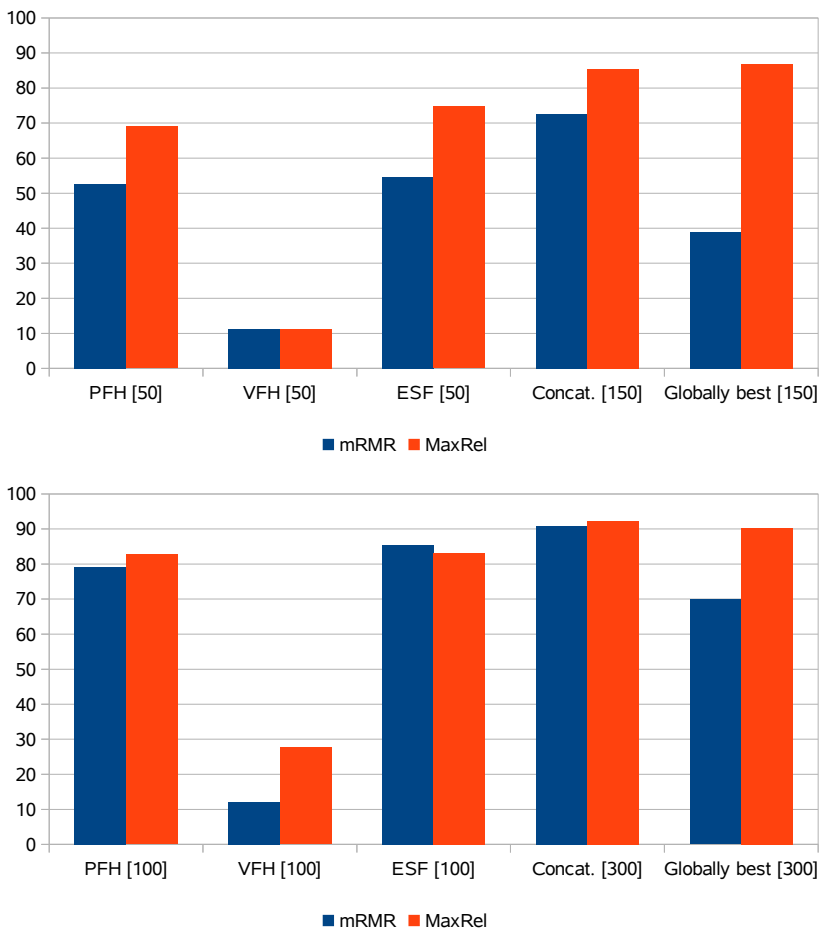

Fig. 7. Linear SVM 5-fold cross-validation accuracies on the RGB-D Object Dataset using the 50 (top) or 100 (bottom) highest scoring dimensions of the three descriptors separately, compared to their concatenation and the globally best 150 or 300 dimensions, respectively.

them. This was compared to the globally best 150 or 300 features, respectively, as shown in Figure 7.

We can see that the "balanced" concatenation of top scoring features performs similarly to the globally best features, and in the case of the less optimal mRMR methods, it is even capable of correcting its shortcomings. In general, however, the global scoring of features (i.e. all of them scored together at once) is sufficient.

As our goal is to evaluate how well do the features and training database generalize to a new dataset and acquisition method, we used the models trained on the RGB-D dataset from Lai et al. for categorizing the object scans we created

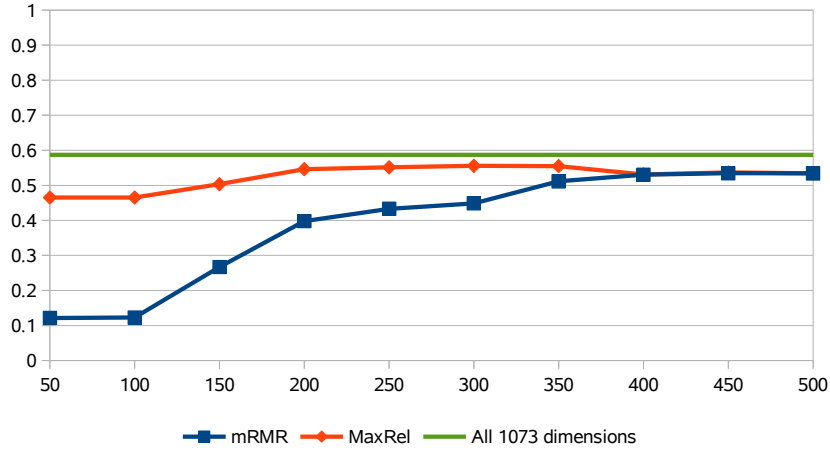

Fig. 8. Linear SVM prediction accuracies on the DLR dataset using the concatenation of all three descriptors. The top scoring feature dimensions according to mRMR and MaxRel were tested in steps of 50 up to 500, and compared to the case when every feature dimension is kept.

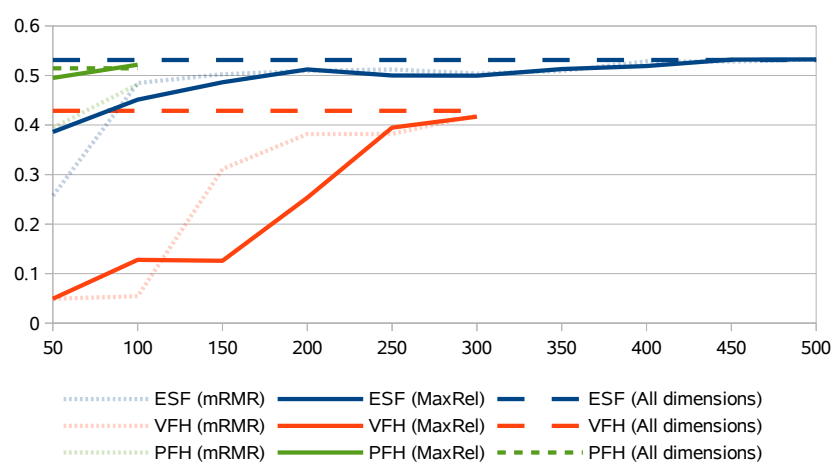

Fig. 9. Linear SVM prediction accuracies on the DLR dataset using the three descriptors separately. The top scoring feature dimensions according to mRMR and MaxRel were tested in steps of 50, and compared to the case when every feature dimension is kept.

at DLR.

The results highlight the difficulties posed by domain change, due to the limited generalization power of the training data and features, reaching an accuracy of approximately $60 \%$. This is in strong contrast to the randomized cross validation results presented above, as shown in Figures 8, 9 and 10.

Thus, we can safely conclude that some sort of domain adaptation is required. Instead of a weighting approach performed in [12], we left one object instance per category out of our testing dataset, and included them during training. To compensate for the added training examples, one of the original training instances was left out of training. We then compared the results on the remaining objects obtained using the original and the updated model, shown in Figure 11.

The adaptation step was performed multiple times in a randomized way in a form of jackknifing, and the minimal and maximal accuracies shown by the error bars. The baseline of $60.62 \%$ using the original model is based on a single classification of the DLR objects, where one object per category was removed (which produced a negligible improvement with respect to Figure 8). However, based on the randomized results for the updated model (and those that 

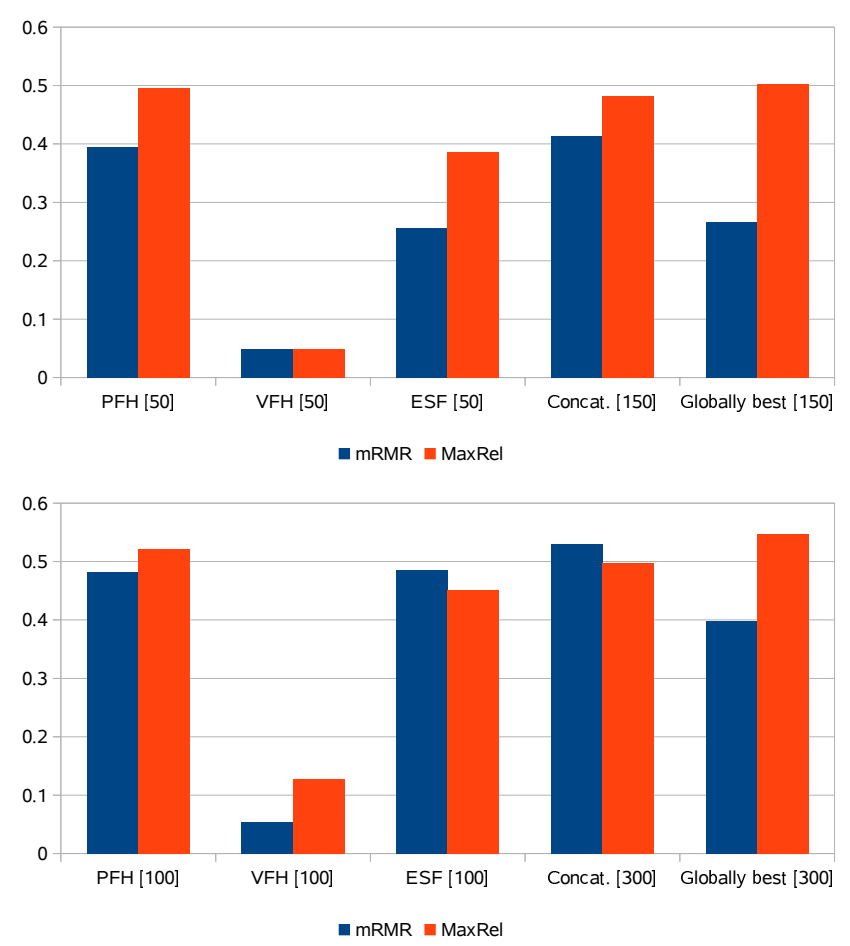

Fig. 10. Linear SVM prediction accuracies on the DLR dataset using the 50 (top) or 100 (bottom) highest scoring dimensions of the three descriptors separately, compared to their concatenation and the globally best 150 or 300 dimensions, respectively.

will be presented in Figure 12), we don't expect a deviation larger than a few percents for this case either.

Even this simple mixing of domains resulted in an improvement to an average of $70 \%$ in the categorization accuracy on the new domain's objects. In total around 15000 RGB-D point clouds from the original training domain were replaced by around 2500 from the new one, however, the original clouds had large overlaps (the authors proposed using every fifth scan only). As there are on average around 5-6 object instances per category, the data from the new domain constitutes only a small portion of the updated dataset, but it was sufficient for improving the results considerably. The parameters obtained by cross-validation on the original dataset were reused, so the re-training lasted only 4.5 minutes.

In case all the original objects were kept for the updated training dataset, the accuracy was $69.41 \%$, thus the accuracy increase was not due to leaving bad data out of the training set. For an in-depth analysis of the variability in object categories that is captured by the RGB-D Object Dataset, we performed another set of randomized experiments (10 runs each), as shown in Figure 12. First, in the left part, we can see that reducing/increasing the number of objects in the source dataset does not affect the general accuracy, but there are clearly objects that are more similar to testing ones than others. As the number of objects increase, their effect diminishes, and the variance between different splits decreases considerably. Second, on the right, the effect of
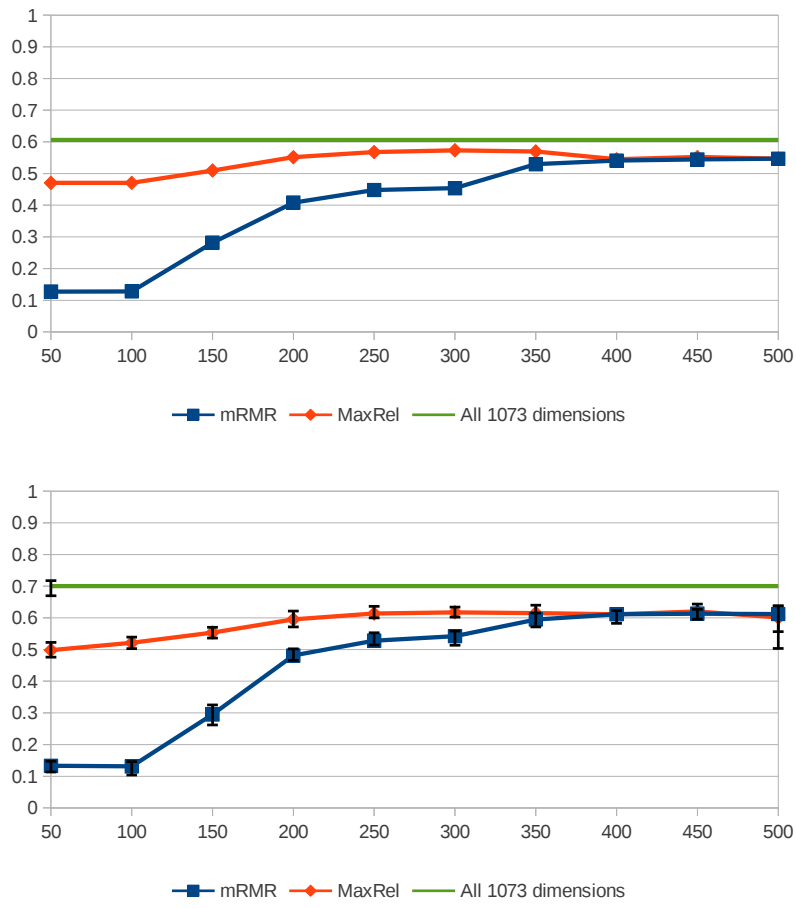

Fig. 11. Linear SVM prediction accuracies on the DLR dataset using the concatenation of all three descriptors, with one object left out per category. Top: the original model trained on the RGB-D Object Dataset was used Bottom: the model was re-trained by exchanging one object per category from the original training data with the objects left out from the testing dataset. Error bars show the minimum and maximum accuracies obtained using all features (10 runs), and each feature selection step (5 runs).
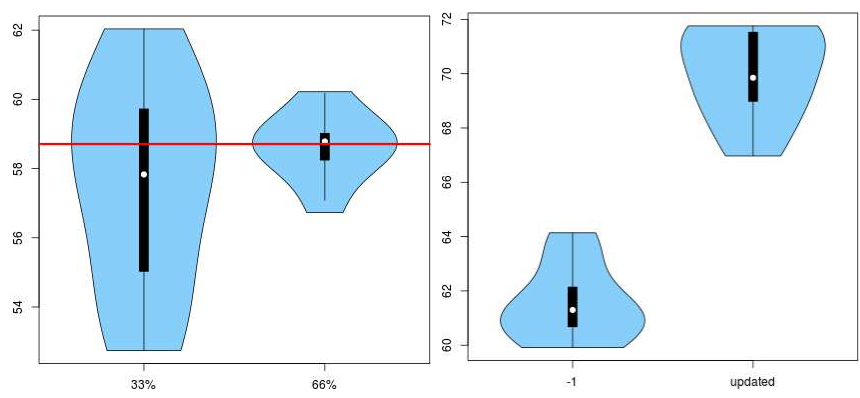

Fig. 12. Left: distribution of the accuracies on the full DLR dataset, when one or two thirds of the object instances are used (the red line shows the result for 100\%). Right: clear improvement on the reduced DLR dataset ( 1 object per category left out for adaptation) when the training dataset is adapted, with respect to using only objects from the RGB-D Object Dataset.

adding an object from the new domain is made apparent: when random objects are added to the training set there is a consistent performance increase (in each run we test the same original training objects and testing objects, once without and then with adaptation).

Similarly to the left part of Figure 12, a reduction in variance can be seen in Figure 13, where the two feature selection methods are evaluated. Their performance follows the same general path than when using $100 \%$ of the object instances, with $66 \%$ of the data already showing relatively stable results, suggesting that there might be some redun- 


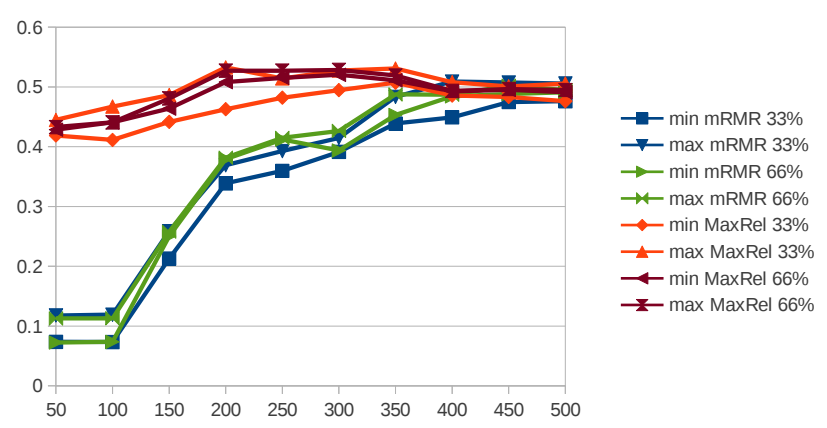

Fig. 13. Results using only a subset of the features, showing the minimum and maximum of the accuracies obtained on the DLR dataset, when one or two thirds of the object instances are used.

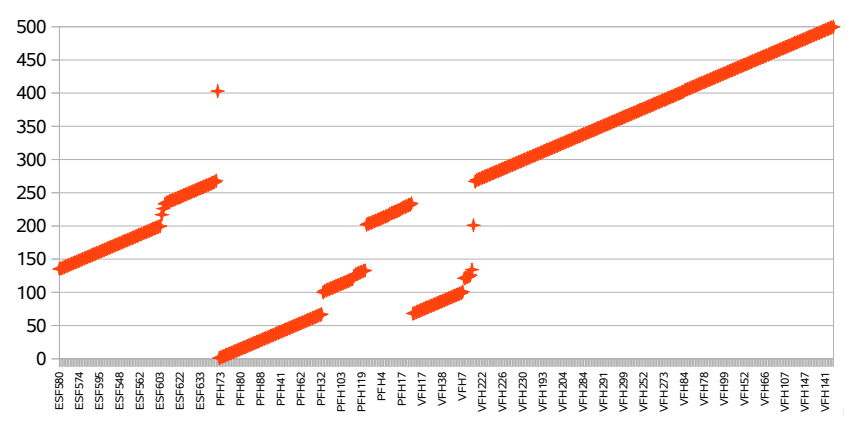

+ Order according to MaxRel

Fig. 14. The ordering of the best 500 feature dimensions according to MaxRel, shown for the three descriptors consecutively. First part shows the ESF dimensions' ordering, the second the positions of the PFH dimensions in the ordering, and finally, the VFH dimensions' positions.

dancy in the training set.

\section{Discussion on Feature Relevance}

As noted above, the ordering of the feature dimensions according to the MaxRel algorithm provided an good indication of which of them holds the most discriminative power. Therefore we analyzed which descriptors, and what part of them are most useful.

Looking at the ordering provided by MaxRel (overview shown in Figure 14), we can see a clear clustering of descriptor dimensions. The best 67 dimensions are all from $\mathrm{PFH}$, then followed by the remaining PFH dimensions mingled with some of the VFH and most ESF ones. The last dimensions from the top 500 are all from VFH, except a single ESF value.

As all of the PFH dimensions are included in the top 250, and the classification accuracies using them are already quite close to the maxima, we can conclude that the information captured by PFH is the most relevant for this categorization task. It has only 125 dimensions, which makes training models fast, however, it has a combinatorial runtime complexity. Nonetheless, it was already used for categorization tasks [20], being able to distinguish 15 (view dependent) surface types.
The PCL implementation computes three angles based on a point-pair's normals $(\alpha, \phi, \theta)$, divides the range of their values in 5 intervals, and creates histogram bins that correspond to specific value combinations. The top 67 dimensions are all histogram bins (feature dimensions) from the range $[32,99] \backslash\{31\}$ (with 31 being ranked at position 100), meaning that the mid-range values of the angles are most discriminative (intervals 2-3 for $\alpha$ and $\theta$, and 2-4 for $\phi$ ). This makes sense, because we use PFH as a global descriptor, capturing the variability of estimated surface normal angles to distinguish objects of different categories. In such a task, extreme values of angles correspond to normals pointing in the same or opposite directions. The latter is quite rare in RGB-D images, and the former does not have much discriminative value. The use of PFH as a global descriptor corresponds to its original design, as conceived at DLR [21], only with one of the constituent features (point-to-point distance) left out.

The usefulness of these normal angles is further highlighted by the results obtained by VFH. This descriptor has two parts, one based on the fast variant of PFH (FPFH), and another that is viewpoint dependent. Overall, VFH performs very well, both for pose estimation and categorization [4], but the highest ranking dimensions come all from the PFHbased part of it. This makes sense, as the view-variant part is not so useful for categorization, and thus those dimensions are ranked at the lower end of the top 500 .

The ESF dimensions that made it to the top 500 are all from the range [537,640], with only dimension 539 missing, and 537 being the one not part of the main cluster, at position 403. Because ESF is a concatenation of ten 64-bin histograms, this range corresponds to the ones for the shape functions 8 (second half) to 10 as being most important for object categorization. These are all point pair (line) features, describing the lines which cross unoccupied space in the point cloud. A similar approach to quantify the amount of free space through point-to-point line traces is also the idea behind GFPFH, the global feature that integrates besides this information also local shape classification using FPFH [4].

While it is clear that MaxRel produces a better feature ordering for our object categorization task, its absolute correctness is difficult to asses. Since SVM are not affected by the Hughes phenomenon, the occasional dips in performance as the number of dimensions increases suggests that some of the features are wrongly selected. Figure 15 shows the features' performance in groups of 50, where a downward trend would be expected if they are sorted correctly. These combinations consider correlations between features as well, so high values are not so surprising also using lower ranked features. However, mRMR has a clearly inverted trend, and considering the relation between the mRMR and MaxRel ordering in Figure as well, it is safe to conclude that MaxRel's ranking is more reliable. Moreover, the selected feature ordering seems to generalize well to a new dataset, as there is a very high correlation in feature performance on the source and target domain ( 0.99 for mRMR and 0.97 for MaxRel). On average around 60\% of the accuracy is 


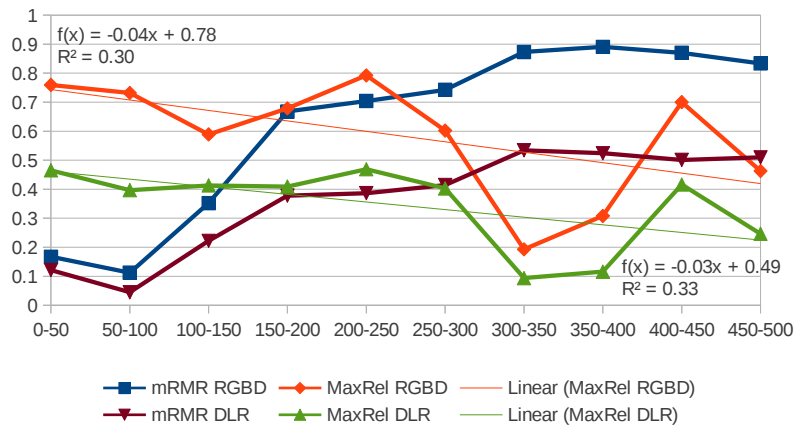

Fig. 15. Feature combination accuracies in groups of 50, as sorted by mRMR and MaxRel, both on the training data (RGBD) and on the target data (DLR). The decreasing linear fit is shown for MaxRel, suggesting that the ordering it produced is correct.

preserved by the feature combinations when dealing with the new object instances.

\section{CONCLUSION}

In our evaluations we have quantified and discussed the effect of training object category recognizers on one dataset and testing it on another one, captured in a different environment, and slightly different conditions. The results show that even the largest RGB-D training database available online does not capture a sufficiently high variation in common object categories, and that some form of domain adaptation is needed. Even a simple approach proved to be highly effective, and could be improved further by methods presented in [22] for avoiding the costly re-training step, by updating existing models with new data.

Based on the comparison of features, and the useful insights given by MaxRel, we can conclude that a wellchosen subset of features is already capable of capturing most of the information necessary for the presented categorization task involving 21 object categories. However, as we saw, the real-world variance in object categories can be quite difficult to capture in current datasets, and there are thousands of object categories of potential relevance [23]. Nonetheless, identifying the most relevant parts of long feature vectors, at least for a given limited application, can aid in optimizing performance, and also to guide future descriptor designs to focus on the most descriptive properties.

\section{ACKNOWLEDGEMENTS}

The authors would like to thank Jorge Nuricumbo Morales for his help with the dataset, and the three anonymous reviewers for their helpful comments.

\section{REFERENCES}

[1] Kevin Lai, Liefeng Bo, Xiaofeng Ren, and Dieter Fox, "A large-scale hierarchical multi-view rgb-d object dataset.," in ICRA. pp. 18171824, IEEE.

[2] Haider Ali, Faisal Shafait, Eirini Giannakidou, Athena Vakali, Nadia Figueroa, Theodoros Varvadoukas, and Nikolaos Mavridis, "Contextual object category recognition for RGB-D scene labeling," Robotics and Autonomous Systems, vol. 62, no. 2, pp. 241 - 256, 2014.

[3] "Microsoft kinect. http://www.xbox.com/en-us/kinect," .
[4] Aitor Aldoma, Zoltan-Csaba Marton, Federico Tombari, Walter Wohlkinger, Christian Potthast, Bernhard Zeisl, Radu Bogdan Rusu, Suat Gedikli, and Markus Vincze, "Tutorial: Point cloud library: Three-dimensional object recognition and 6 dof pose estimation.," IEEE Robot. Automat. Mag., vol. 19, no. 3, pp. 80-91, 2012.

[5] Hanchuan Peng, Fuhui Long, and Chris Ding, "Feature selection based on mutual information: criteria of max-dependency, max-relevance, and min-redundancy," IEEE Transactions on Pattern Analysis and Machine Intelligence, vol. 27, pp. 1226-1238, 2005.

[6] Wei Zhang, G. Zelinsky, and D. Samaras, "Real-time Accurate Object Detection using Multiple Resolutions," in Computer Vision, 2007. ICCV 2007. IEEE 11th International Conference on, 2007, pp. 1-8.

[7] Stephen Gould, Tianshi Gao, and Daphne Koller, "Region-based segmentation and object detection," in Advances in Neural Information Processing Systems 22, Y. Bengio, D. Schuurmans, J. Lafferty, C. K. I. Williams, and A. Culotta, Eds., pp. 655-663. 2009.

[8] Alexander Toshev, Ben Taskar, and Kostas Daniilidis, "Object detection via boundary structure segmentation," in CVPR, 2010, pp. 950-957.

[9] Zheng Song, Qiang Chen, ZhongYang Huang, Yang Hua, and Shuicheng Yan, "Contextualizing object detection and classification," in CVPR, 2011, pp. 1585-1592.

[10] K. Lai and L. Bo and X. Ren and D. Fox, "Detection-based Object Labeling in 3D Scenes," in IEEE International Conference on on Robotics and Automation, 2012.

[11] Nicolas Burrus, Mohamed Abderrahim, Jorge Garcia, and Luis Moreno, "Object reconstruction and recognition leveraging an rgb-d camera," in MVA IAPR Conference on Machine Vision Applications, June 13-15 2011, pp. 132-135.

[12] Kevin Lai and Dieter Fox, "Object recognition in 3d point clouds using web data and domain adaptation," Int. J. Rob. Res., vol. 29, no. 8, pp. 1019-1037, July 2010.

[13] L. Bo, X. Ren, and D. Fox, "Unsupervised Feature Learning for RGBD Based Object Recognition," in ISER, June 2012.

[14] Manuel Blum, Jost Tobias Springenberg, Jan Wulfing, and Martin Riedmiller, "A learned feature descriptor for object recognition in rgb-d data.," in ICRA. 2012, pp. 1298-1303, IEEE.

[15] R.B. Rusu and S. Cousins, "3d is here: Point cloud library (pcl)," in Robotics and Automation (ICRA), 2011 IEEE International Conference on, may 2011, pp. $1-4$.

[16] Zoltan-Csaba Marton, Ferenc Balint-Benczedi, Oscar Martinez Mozos, Nico Blodow, Asako Kanezaki, Lucian Cosmin Goron, Dejan Pangercic, and Michael Beetz, "Part-based geometric categorization and object reconstruction in cluttered table-top scenes," Journal of Intelligent and Robotic Systems, pp. 1-22, 2014.

[17] K. Lai, L. Bo, X. Ren, and D. Fox, "A large-scale hierarchical multiview RGB-D object dataset," in Proc. Int. Conf. on Robotics and Automation, Shanghai, China, May 2011, pp. 1817-1824.

[18] Zoltan-Csaba Marton, Florian Seidel, Ferenc Balint-Benczedi, and Michael Beetz, "Ensembles of Strong Learners for Multi-cue Classification," Pattern Recognition Letters (PRL), Special Issue on Scene Understandings and Behaviours Analysis, 2012, In press.

[19] Chih-Chung Chang and Chih-Jen Lin, "LIBSVM: A library for support vector machines," ACM Transactions on Intelligent Systems and Technology, vol. 2, pp. 1-27, 2011.

[20] Radu Bogdan Rusu, Zoltan Csaba Marton, Nico Blodow, and Michael Beetz, "Learning Informative Point Classes for the Acquisition of Object Model Maps," in Proceedings of the 10th International Conference on Control, Automation, Robotics and Vision (ICARCV), Hanoi, Vietnam, December 17-20, 2008.

[21] Eric Wahl, Ulrich Hillenbrand, and Gerd Hirzinger, "Surflet-pairrelation histograms: A statistical 3d-shape representation for rapid classification," in 3D-Digital Imaging and Modeling (3DIM), Banff, Canada, October 2003.

[22] Jun Yang, Rong Yan, and Alexander G. Hauptmann, "Cross-domain video concept detection using adaptive svms," in Proceedings of the 15th International Conference on Multimedia, New York, NY, USA, 2007, MULTIMEDIA '07, pp. 188-197, ACM.

[23] Irving Biederman, "Recognition-by-components: A theory of human image understanding," Psychological Review, 1987. 